
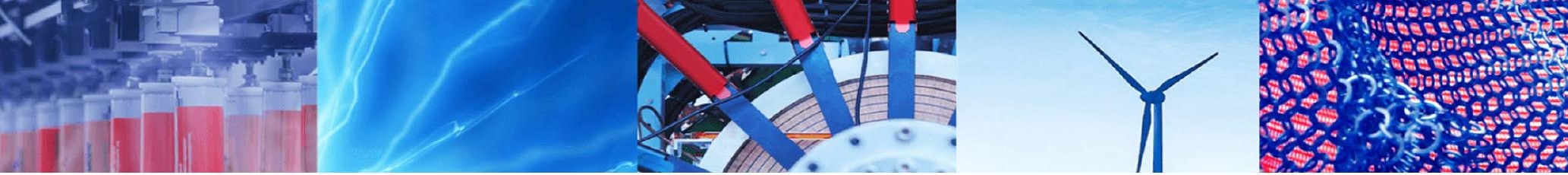

Research Article

\title{
Route optimization for warehouse order picking operations via vehicle routing and simulation
}

\author{
Namrata Shetty ${ }^{1} \cdot$ Bhawesh Sah $^{2} \cdot$ Sung Hoon Chung ${ }^{2}$ (D)
}

Received: 22 August 2019 / Accepted: 21 January 2020 / Published online: 31 January 2020

(c) Springer Nature Switzerland AG 2020

\begin{abstract}
Order picking has been recognized as one of the most challenging activities in terms of time, labor, and cost for most warehouses. Order fulfillment has a definite time constraint that is predefined by the customer and any deficit in the process of order picking at the warehouse level will impact the entire supply chain. This paper addresses the problem of routing optimization for order picking in a warehouse to minimize the travel time and distance. In particular, we propose an easy-to-implement vehicle routing based approach in conjunction with the distance matrix for obtaining an optimal route for order picking, which is solved using the off-the-shelf solver Gurobi with the Julia programming language. The optimal route is then compared with the routes obtained by other traditional approaches such as S-shape, return, midpoint, and largest gap methods by means of simulation. Two order picking demand scenarios are considered: one with uniform pick locations throughout the warehouse and the other with differentiated pick locations. We show that the proposed vehicle routing based approach outperforms the S-shape, return, mid-point, largest gap methods by $36.55 \%$, $44.89 \%, 46.64 \%$, and $36.9 \%$, respectively, for scenario 1 , and by $25.12 \%, 24.01 \%, 27.57 \%$, and $26.77 \%$, respectively, for scenario 2 . The detailed discussion about the routing policy implications on management is also provided.
\end{abstract}

Keywords Vehicle routing $\cdot$ Order picking $\cdot$ Warehouse $\cdot$ Supply chain management $\cdot$ Simulation

\section{Introduction}

Warehousing is an essential part of supply chain management because of its important duties such as storing parts and materials as well as finished products and providing a streamlined, unified means to consolidate materials from suppliers all around the world [11]. In order to keep up with the rising competition in the industry and outperform competitors, warehouses must perform all their operations, e.g., receiving, put-away, cross-docking, order picking, and shipping in the most efficient way to ensure smooth functioning of the supply chain while minimizing the cost.

Among all the warehouse operations, we focus on order picking because it is known to be extremely challenging in terms of labor, time, and cost, which takes $50-75 \%$ of total operating cost for a typical warehouse $[9,16]$. The recent dramatic growth of e-commerce gives rise to the exponential increase of order picking operations $[9,36]$ and, therefore, it has received a considerable attention from both academia and industry [9, 25-27, 30]. Note that among all the activities related to order picking, the picker travel takes the greatest portion of the time spent, followed by search, pick, setup, and other activities as shown in Fig. 2. Accordingly, we aim to contribute to the warehouse operation efficiency by minimizing the order picking travel time and distance by means of the proposed vehicle routing and distance matrix based approach.

This type of problem, to minimize the order picking travel time, is called the picker routing problem (PRP) and

$\triangle$ Sung Hoon Chung, schung@binghamton.edu | ${ }^{1}$ The UPS Store Inc., San Diego, USA. ${ }^{2}$ Department of Systems Science and Industrial Engineering, Binghamton University, Binghamton, USA. 
it is usually formulated as a Steiner traveling salesman problem (TSP) [9], a special case of TSP. In a TSP, each node (geographical locus, e.g., city) is allowed to be visited only once. In a Steiner TSP, however, some nodes are allowed to be visited multiple times while other nodes need not be visited. These special conditions are required for PRP due to the interpretation of the layout of a warehouse to a graph. Figure 1 shows such interpretation where black dots in the right side denote picking locations or a depot and white dots represent cross points between aisles or a corner. Black dots must be visited (and allowed to be visited multiple times) but white dots may or may not be visited. As Steiner TSP is not solvable in polynomial time in general [9], a variety of heuristics and meta-heuristics have been developed [see, e.g., 6, 7, 30].

In this paper, we propose a simple but effective approach that is based on a vehicle routing problem (VRP) formulation and the distance matrix that can be created by some preprocessing and precalculation. That is, we address the PRP as a VRP, unlike other studies that use the Steiner TSP as the model basis. In particular, we employ the Miller-Tucker-Zemlin (MTZ) formulation [32] of the VRP with no complicated constraints such as time windows and no special conditions. Naturally, the VRP formulation can handle the capacity of order pickers, which is a big plus over the Steiner TSP. In our VRP formulation for the PRP, all nodes must be visited only once, which is exactly the same as the conventional TSP or VRP requirements. There is no need to consider some nodes that can be visited multiple times or other nodes that need not be visited. That is, our VRP formulation eliminates the complexity of the Steiner TSP, and such simplification will be made possible by means of the distance matrix that will be introduced in the subsequent section. In summary, the primary advantage of our proposed VRP approach over the Steiner TSP to address the PRP is twofold. First, we can address the capacity of order pickers by means of the VRP formulation. Second, the implementation of the VRP is straightforward, easy, and computationally tractable. This will be particularly useful for practitioners in a warehouse.

\subsection{Background}

Warehouse operations include receiving and unloading inbound materials, putting them away into appropriate storage locations, picking the required materials based on customer orders, and dispatching them to the customer. Any compromise in the performance of any one of these aspects can affect the overall productivity of the warehouse and eventually impact the supply chain as a whole. In fact, material handling along with inventory management is one of the most critical components in supply chain and logistics management.

In this paper, among other types of warehouse we focus on a typical warehouse such as the manufacturing supporting warehouse that houses spare parts and materials necessary for production and the order fulfillment

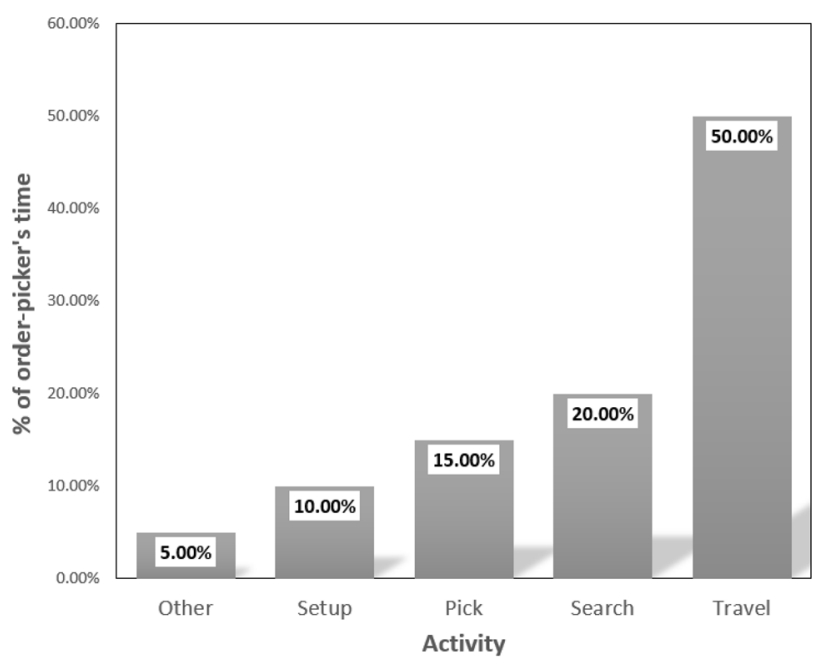

Fig. 2 Time spent by an order picker. Source: De Koster et al. [9]
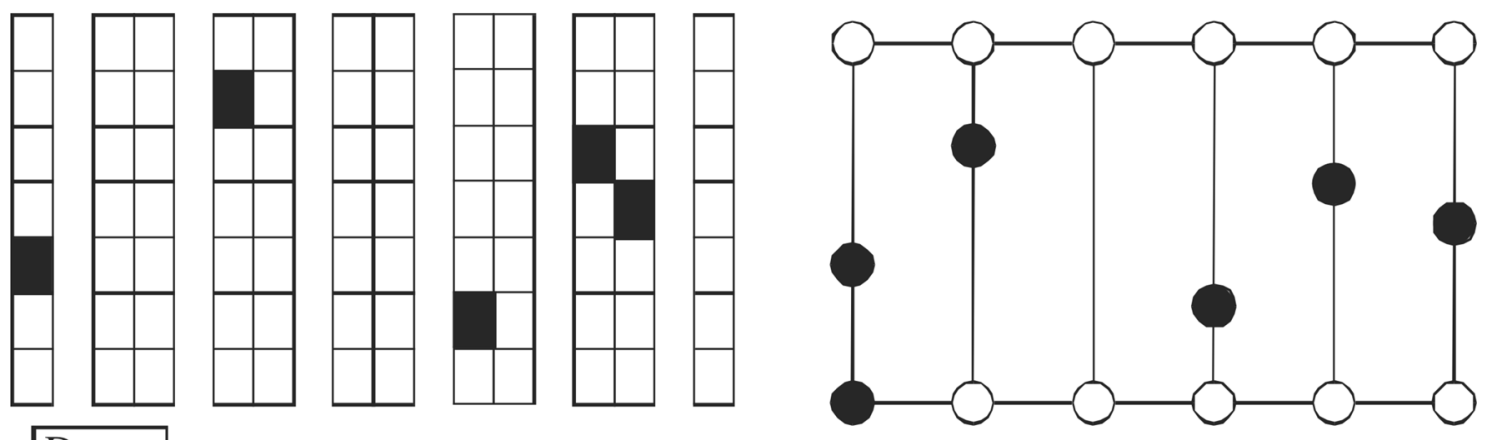

Depot

Fig. 1 Interpretation of a warehouse layout to a graph. Source: De Koster et al. [9] 
warehouse that receives and consolidates a variety of items from different sellers worldwide and ships the items to the customers. However, the proposed methodology can be easily applied to other types of warehouses such as the food storing warehouse that requires temperaturecontrolled zones because our proposed method is based on vehicle routing models that support various types of problems. In a typical warehouse, the inbound and outbound operations take place on either sides of the warehouse and a unidirectional or a bidirectional flow of material is ensured by the warehouse policy. Materials are received by the warehouse from various suppliers around the world and then put-away into storage locations. These locations include high racks for small- to medium-sized parts and floor locations for major mechanical parts. In general, parts with higher demand from the customer are assigned to locations near the outbound side of the warehouse, while the parts with lower demand from the customer are assigned to locations on the far end of the warehouse. This storage policy is motivated by the fact that order picking is one of the most crucial activities, which also has a limited time window. Indeed, storage locations for materials and parts can be decided by a variety of methods such as based on the type of materials and operation characteristics [20]. For example, the relationship between storage policy and order picking travel time and distance has been studied where travel congestion is explicitly considered [20].

In the warehouses considered in this paper, customer demands are received in the form of Kanban triggers for manufacturing supporting warehouses, which need to be fulfilled before the start of shift at the production plant. The order fulfillment warehouses also work in a similar fashion with the limited deadlines for order picking. This requires a high degree of accuracy and speed from the outbound team, especially the order pickers. Along with the time constraint, another important constraint for order picking is the size of the demand. The demand size varies through peak and non-peak seasons. Hence, order picking has to be not only efficient but also robust enough to provide adequate and acceptable outcomes. In this regard, the order picking policy called wave picking can be considered where the frequency, timing, and size of order picking are to be optimized $[5,13,14]$. In order to ensure efficient resource utilization and timely fulfillment of customer demand, the order picking problem in general and the routing optimization in particular are a point of focus in this paper.

\subsection{Order picking and pick path optimization}

Order picking is one of the most crucial activities associated with warehousing and material handling $[9,36]$, which involves retrieving stored parts, materials, and/or partially finished products in order to fulfill a customer demand. This activity heavily impacts the warehouse operations and eventually the performance of the entire supply chain, and it has been identified as one of the most laborious and time consuming activities of warehouse operations. Furthermore, new trends in manufacturing and distribution such as just-in-time manufacturing and same-day shipping, and fast growing e-commerce have made order-picking more complex and difficult to manage. In order to be able to serve customers efficiently, it is vital that the process of order picking be optimized.

Order picking can be categorized into several ways on the basis of the methods that are used to organize and sequence the orders. There are varying degrees of automation involved in the different methods of order picking. This study focuses on manual order picking systems (which involves human operation/not fully automated). The different order picking systems are: picker-to-part, part-to-picker, and put systems. Picker-to-part systems involve the order picker traversing through the warehouse by means of material handling equipment such as forklifts or reach-trucks, physically picking items from their locations to fulfill customer orders. This is the most common method for order picking, which is the focus of this study. Part-to-picker systems involve automated storage and retrieval systems that pick the loads from their locations and bring them to the depot. The primary difference between picker-to-part and part-to-picker systems is that in the former, the picker moves from location-to-location, picking items, whereas in the latter, the picker stays in one location and the items come to him/her. In put systems, the items are retrieved by one of the two above mentioned policies and then distributed into bins by customer. These systems are capable of meeting large number of customer demands within relatively short spans of time [9].

Another important factor that affects the method of order picking is the layout and zones of the warehouse. Items can be zoned if they have special storage requirements such as temperature, humidity, size, weight, etc. Another way of zoning would be by demand of the product where the high demand products are stored closer to the outbound drop off locations, whereas slow moving items are stored further away from the drop off locations. In such cases, order pickers may be confined to an area, and picking items from their respective zones.

Based on the discussion above, we claim that the order picking optimization methods can be largely divided into two categories: pick policy optimization and routing optimization. For a pick policy optimization survey, Bartholdi and Hackman [1] classify order picking operations on the basis of various pick policies: strict order picking, batch picking, zone picking, and wave picking. In the strict 
order picking, a picker traverses the complete route and retrieves all the required materials for order completion while the batch picking involves the completion of multiple orders at the same time. In the zone picking, the warehouse is divided in multiple zones with dedicated pickers assigned to each zone to reduce travel time and distance. However, zone picking may reduce the number of items picked by the pickers. The wave picking policy involves processing and combining orders to be transported in one shipment all at once regardless of zone or location, instead of sending pickers as soon as an customer demand arrives. It aims to take advantage of economies of scale in picking operations, and the topics such as optimal frequency, timing, and size of wave release have been studied $[5,13,14]$. Note that these picking policies can be implemented in various combinations based on requirements.

When an order information including the item description, quantity, and cutoff time is received from a customer and once the picking policy is determined, order pickers using material handling equipment such as forklifts are dispatched to pick the items involved in that order. This is the stage where the routing optimization comes into play to minimize the distance traveled, thus reducing the time and cost of the order picking process as a whole. In this paper, the routing problem has been addressed as a special case of the vehicle routing problem (VRP). The VRP model is formulated and then implemented using the off-the-shelf solver in conjunction with Julia programming language in order to obtain the shortest path for order picking, such that the travel distance of the material handling equipment can be minimized. Furthermore, the optimal solution is then compared with routes obtained by other traditional routing policies such as S-shape, return, mid-point and largest gap methods by means of simulation and statistical analysis. Visual Basic Application (VBA) is used for modeling and simulation. Simulation has been widely used for the analysis and optimization of supply chain and logistics operations, [see, e.g., 2, 4, 19, 26, 35].

\subsection{Motivation and objective of the study}

A literature review of relevant work reveals that a considerable amount of research has been conducted with a focus on vehicle routing in the field of supply chain and logistics. While most research in the literature develops a new mathematical model and relevant methodology to tackle the transportation problem in supply chain and logistics, this paper employs both mathematical model and simulation to focus on the order picking routing problem in a warehouse setting with the goal of optimizing the process and improving overall productivity. The objective is to minimize the total distance and travel time for order picking operation by material handling equipment, thus minimizing the overall time taken for and costs associated with order picking. In more detail, this paper aims to achieve the following:

1. Generating a mathematical model for routing with a consideration of various constraints in a warehouse order picking operation.

2. Implementing the mathematical model to provide an optimal picking route.

3. Performing simulation-based analyses and compare the mathematical model results with alternative picking policies.

4. Suggesting the order picking approach that is simple to implement with adequate outcomes.

The remainder of this paper is organized as follows. Section 2 highlights the existing research and solutions towards solving the order picking problem. Section 3 illustrates the methodology including the description of the system under study. It introduces and explains the mathematical model developed and implemented to solve the problem. Section 4 presents the results and discussion. Finally, Sect. 5 concludes the paper and discusses the potential future research directions.

\section{Literature review}

A significant amount of studies have been conducted in the domain of routing policies for various warehouse layouts as well as in the area of vehicle routing. With respect to a literature survey, [9] provide an overview on characteristic decision problems faced in the design and control of manual order-picking processes. This paper identifies order picking as laborious in terms of cost, time, and man-power, and describes the complexity of order picking systems as shown in Fig. 3. In warehouse operations and management, routing, storage, batching, zoning, and order release mode are in the policy level while warehouse dimensionality, information availability, mechanisation level, and command cycle are in the strategy level. It also explicates various order picking methods and summarizes some of the established routing heuristics such as S-shape, return, mid-point and largest gap policies.

A variety of methods have been proposed for the routing optimization in a warehouse setting. For example, mathematical optimization approaches $[10,28,31]$ and heuristics $[3,10,29,31]$ are proposed, along with the studies that compare the exact method and heuristics $[29,31]$. For exact method examples, Ratliff and Rosenthal [28] propose an algorithm based on dynamic programming for order-picking in a rectangular warehouse with crossovers only at the end of aisles. Roodbergen and 
Fig. 3 Complexity of order picking systems. Source: [9]

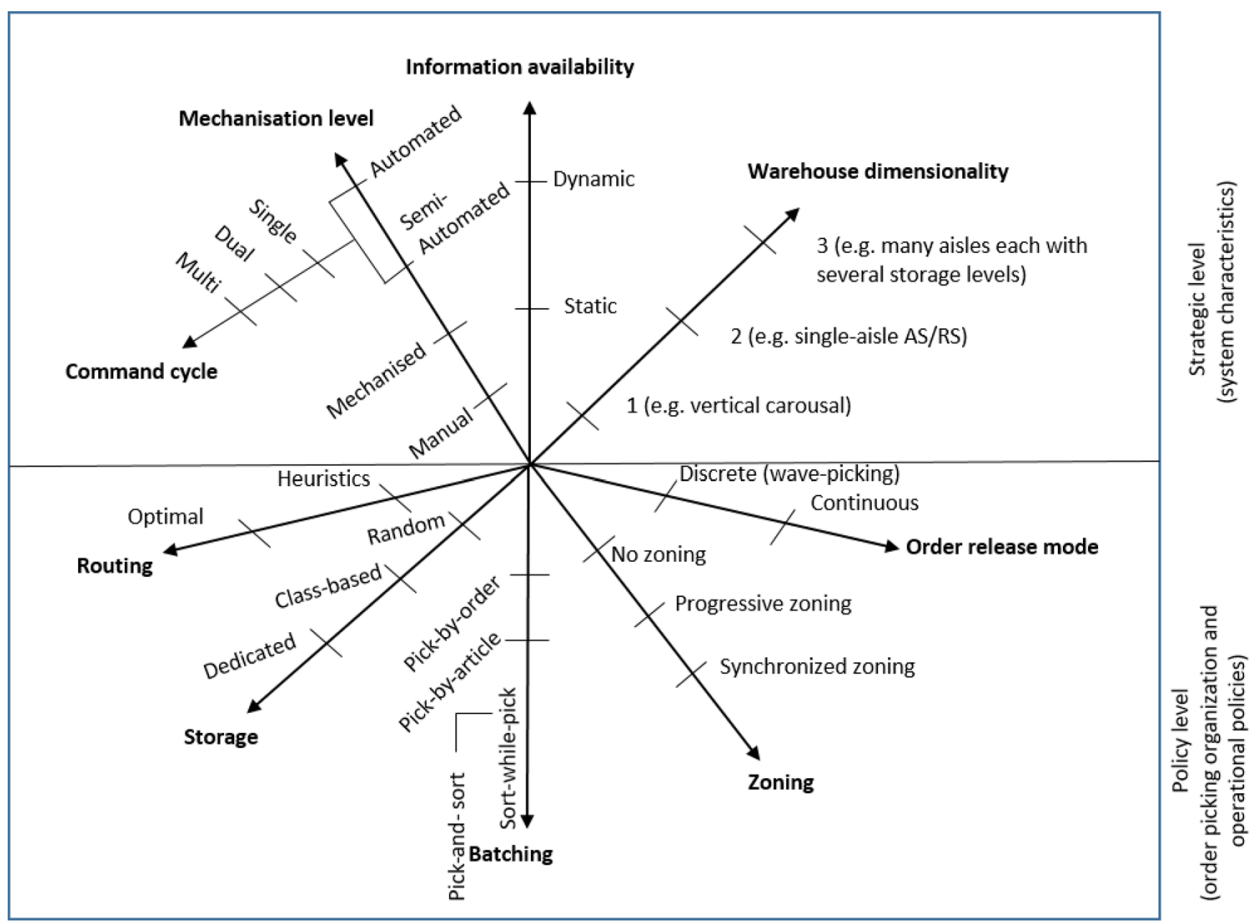

Koster [29] present a branch and bound algorithm for the TSP to find the shortest path in a parallel aisle warehouse with crossovers at the end of every aisle and also halfway along the aisles, which is compared with the routing heuristics such as S-shape, Aisle-by-Aisle, largest gap and a combined method. This paper also discusses some layout consequences, which show that the addition of cross aisles may decrease the overall material handling time by reducing the travel time for picking. With respect to heuristics, Caron et al. [3] evaluate the routing heuristics for a manual picker-to-part system, which use the storage policy based on the cube-per-order index (COI) to ensure that the large and heavy items with high demand are placed as close to the outbound area as possible. Dekker et al. [10] present heuristics for the different order picking policies such as $\mathrm{S}$-shape, mid-point, and Combined in conjunction with the storage policy, as the storage policy is closely related to the routing policy. For the studies that compare the exact methods and heuristics, Theys et al. [31] present an algorithm based on the NP-hard Steiner TSP to deal with the routing problem for order picking in a conventional multiparallel-aisle warehouse. Interestingly, they evaluate the degree to which the TSP-based approach affects the performance improvement as opposed to dedicated heuristics such as S-shape, return, and largest gap. The authors validate whether it is necessary to use complex heuristics for routing of order pickers as compared to simple approaches such as S-shape method.

Turning our attention to the storage policy and warehouse layout, there is also a considerable amount of research conducted because their close relationship to the routing policy and contribution to the overall warehouse operation effectiveness. For example, Hall [15] emphasizes on the impact of warehouse layout on order picking through an analytical approach and Petersen and Aase [26] evaluate the relationship between routing policies and the warehouse layout by simulation. In addition, Dekker et al. [10] put forth different storage policies including the $A B C$ method where all the items to be stored are categorized into $A, B$, or $C$ depending on their demand and turnover rate. As the storage policy eventually has an impact on the order picking route, the preferred routing policy is correlated with the chosen storage method, which is based on the skewness of the ABC-curve. The more skewed this curve is, the higher difference between pick frequencies of A, B, and C items. Gu et al. [14] highlight the impact of the traffic congestion caused by material handling equipment used for order picking such as forklifts and reach-trucks on order picking productivity, and show how the congestion is related to the warehouse layout.

It is worth providing a brief survey on TSP and VRP because these are the basis of our proposed approach to tackle the order picking routing problem. Dantzig and Ramser [8] originally propose and formulate the VRP to minimize the total travel cost, subject to a set of constraints. Ratliff and Rosenthal [28] focus on a special case of the traveling salesman problem with a class of matrices that follow a pattern of Hamiltonian circuits. This is an exemplary work that shows the mathematical approaches involved in TSP and VRP require a heavy 
mathematical calculation, namely an NP-hard problem. Fukasawa et al. [12] highlight the importance of taking into account uncertain demand, traffic conditions, and/ or service times. This study uses the robust optimization method for the VRP under uncertainty. Furthermore, Juan et al. [18] emphasize a particular case of the TSP known as the Vehicle Routing Problem with Stochastic Demand (VRPSD). The Monte-Carlo simulation approach is chosen owing to the random nature of the demand and the possibility of unfeasible solutions when the demand exceeds vehicle capacity. Similarly, a simulation-based algorithm for the capacitated vehicle routing problem (CVRP) with stochastic travel time is presented [34]. For easy implementation, Jiang [17] uses Microsoft Excel Solver to solve the TSP. This paper considers two cases of the TSP, viz. Euclidean Traveling Salesman Problems (ETSP) and the Random Link Traveling Salesman Problems (RLTSP). This paper highlights the pros of using Microsoft Excel to solve routing problems such as the TSP. The work of Toth and Vigo [32] explicates in detail the various types of VRPs, and it is also an excellent textbook for VRP.

In summary, a vast amount of research has been carried out in the field of picking policies, routing policies, and VRP in a warehouse setting. However, the research that evaluates the VRP-based routing policy compared with other heuristics by means of simulation and statistical analysis, which also employs plausible scenarios, is scarce. This research aims to fill such a gap, provide a practical guidance to the industry, and contribute to the literature.

\section{Model and methodology}

This section illustrates the routing model and methodology. First, the system under study is described in detail, followed by the assumptions used in the model. The VRP model is formulated and the alternative heuristics policies are introduced. Furthermore, the simulation approach is explained.

\subsection{System description}

The system under study is a typical warehouse facility, usually run by a third party logistics company, for a manufacturing plant, or functions as a order fulfillment center. The warehouse holds parts needed by the manufacturer in order to assemble and produce the final product, or works as a buffer between suppliers and end customers in the capacity of an order fulfillment center. Warehouses have in general two primary location types: floor locations and rack storage locations. The floor locations are used for unloading area, temporary spaces during the put-away operation, and storage space for bulky and large items such as major mechanical parts, while smaller parts are stored in appropriate containers in the racks. As the rack space takes the vast majority of spaces in most warehouses, the focus of this study is the rack locations. However, the same VRP approach can be applied to the general warehouse storage types including the floor space.

The warehouse we consider in the case study is based on the actual one from a partner company, which has 30 parallel racks having 7 levels, with 24 bay locations for each row, as shown in Fig. 4. Each bay location has 3 bins. Also, levels 5, 6, and 7 have a bay in the middle cross aisle. Hence, there are 25 bay locations for each row for levels 5-7, while there are 24 bay locations for each row for levels 1-4. This layout is a multiparallel-aisle with cross aisles at the middle and both ends of the rack. Materials are stored and retrieved from the bay locations based on the two criteria. First, the location should be able to sustain the size and weight of the material. In accordance with this, heavy materials are stored at the bottom levels and smaller parts are stored in high racks. The second decision factor is based on demand. The high flowing materials are stored towards the front end, near the outbound dock doors while the slow moving materials are stored towards the back end, close to the inbound docks. This implies that the pick locations are more towards the front end and less towards the back end of the warehouse. In this study, to examine the impact of different storage policies, we consider the two scenarios: one with uniform pick up locations throughout the warehouse (for random storage policy) and the other with more pick up locations towards the front side (for demand-based storage policy). The customer requirements are generated through a warehouse management system by means of a batch program in the form of Kanban triggers. The batch program generates these requirements in two batches, once for the day shift and once for the night shift. Based on the production requirements, the number of triggers dropped by the system ranges between 1500 and 3500 per day, and on an average 1500 and 2000 triggers are dropped each day. Pick tickets are generated and printed for each trigger by the system. These pick tickets are then manually sorted based on the destination/consumption point and assigned to the order pickers. Each trigger is fulfilled by picking the material from the location printed on the pick ticket and dropping it off to the assigned outbound area. Each order picker uses the material handling equipment such as a forklift or a reach-truck to pick the items. The order pickers physically pick parts from respective locations and stage them in the staging area before loading them onto trailers, which are then dispatched to the customer. Figure 5 provides a high level flowchart of the order picking process. 
Fig. 4 Layout of racks section
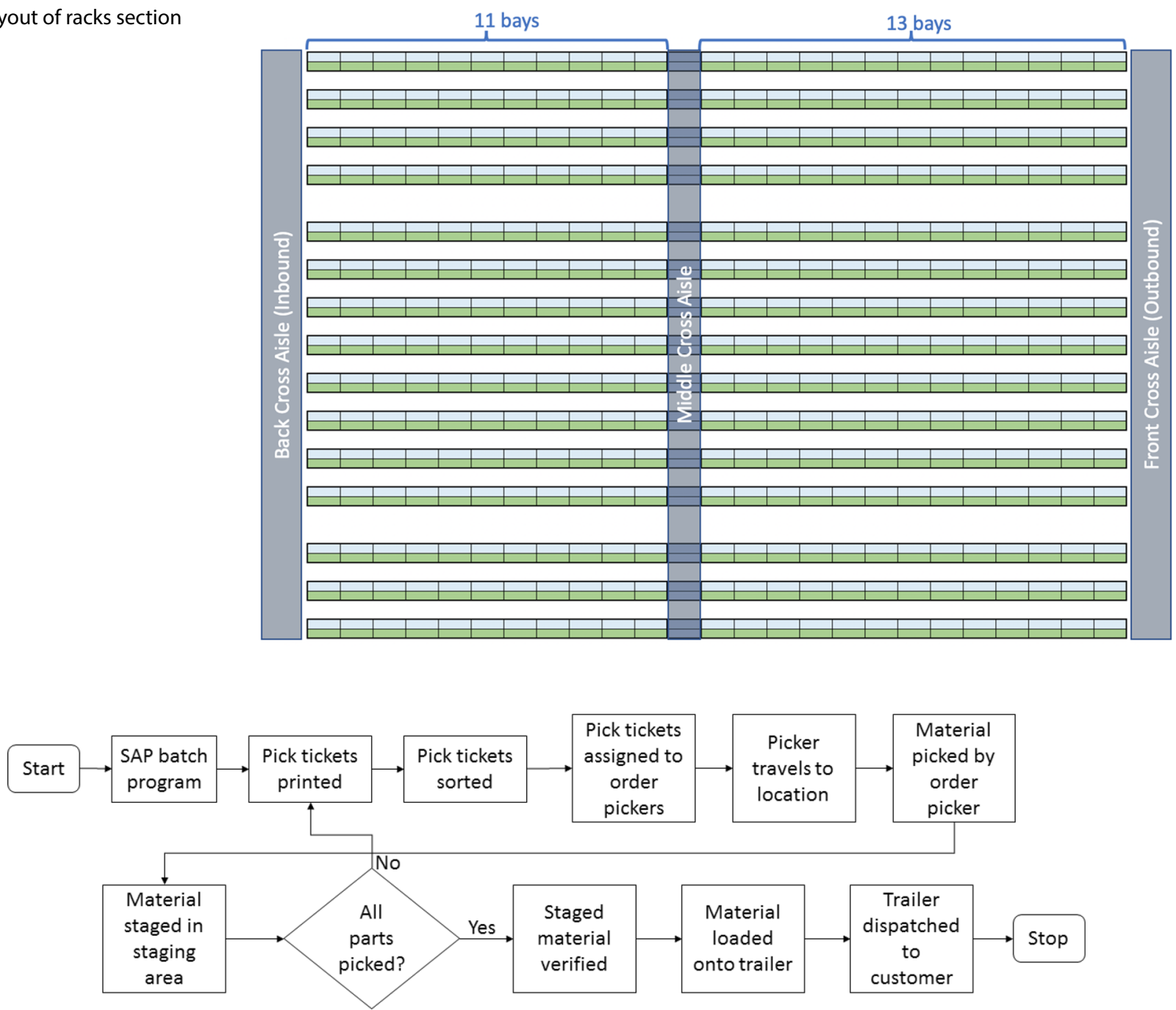

Fig. 5 Order picking process

\subsection{Assumptions}

The proposed model and methodology require certain assumptions to reasonably simplify and appropriately represent the system, achieve computational tractability, and solve the problem. First, it is assumed that the travel path chosen for travel between any two pick locations is always the shortest possible path. Practically, this may be a little arduous to achieve since it would require the order pickers to know the shortest possible route between any two nodes. However, with appropriate visual aid and/or voice aid that can be attached to the material handling equipment, this can be made possible. Indeed, there has been a significant progress for semiautonomous or autonomous material handling equipment such as automatic forklifts employing sensors, RFID and ultra wide band technologies, and adaptive algorithms to enhance safety and productivity of order picking operations [33]. Next, the drop-off location (staging area) is assumed to be at the bottom-right corner of the warehouse for the sake of simplicity. However, this assumption can be easily modified for further analysis. An important assumption is that the size of the items to be picked allows multiple picks on a single tour without exceeding the vehicle capacity. Also, vehicle capacity is assumed to be 14 items for the purpose of calculations, which implies that the number of picks can be up to 14 in a single picking run. This number can be modified for further research. It is also assumed that a single vehicles is used in the case study, which can be updated to accommodate multiple order pickers. Another important assumption is that each pick-up location can be visited only once in a single picking operation. Finally, it is assumed that there are no obstacles on the travel path, thus enabling uninterrupted travel. 


\subsection{Mathematical model}

This section introduces the mathematical formulation of the VRP model that is used in this study. Each pick location is considered to be a node with the drop-off location (defined as a depot) being node 0 . The set of all nodes excluding the depot is denoted by $N=\{1, \ldots, n\}$ and $N_{0}=\{0\} \cup\{1, \ldots, n\}$ is the set of all nodes. Let $i$ and $j$ be the index nodes such that $i, j \in N_{0}$. The vehicle (material handling equipment, e.g., forklift) capacity is denoted by $C$ and the set of vehicles is denoted by $K=\{1,2, \ldots|K|\}$. The travel distance, which can be converted to travel time by assuming the constant average speed of the vehicles, between node $i$ and $j$ is denoted by $t_{i j}$ while the pick quantity or demand at each node $i$ is denoted by $d_{i}$. $T$ is defined as the maximum time allowed for each tour and the flow of the vehicle after visiting node $i$ is denoted by $u_{i}$ while $a_{i}$ represents the arrival time of the vehicle at node $i$. Let the binary variable $x_{i j}$ be an indicator of travel from node $i$ to $j$ ( 1 implying travel between the two nodes; 0 implying otherwise). Now the VRP based on the MTZ formulation [32] can be formulated as follows:

$\min \sum_{i \in N_{0}} \sum_{j \in N_{0}} t_{i j} x_{i j}$

subject to

$$
\begin{aligned}
& \sum_{i \in N_{0}} x_{i j}=1 \quad \forall j \in N \\
& \sum_{j \in N_{0}} x_{i j}=1 \quad \forall i \in N \\
& \sum_{i \in N} x_{i 0}=|K| \\
& \sum_{j \in N} x_{0 j}=|K| \quad \forall i, \quad \forall i \in N \\
& u_{i}+d_{j}-C\left(1-x_{i j}\right) \leq u_{j} \\
& d_{i} \leq u_{i} \leq C \quad \forall i, j \in N \\
& a_{i}+t_{i j}-T\left(1-x_{i j}\right) \leq a_{j} \\
& t_{1 i} \leq a_{i} \quad \forall i \in N \\
& x_{i j} \in\{0,1\} \quad \forall i, j \in N_{0}
\end{aligned}
$$

The objective function (1) is to minimize the total travel distance/time to complete the order picking process while constraints (2) and (3) ensure that each node is visited only once. Constraints (4) and (5) specify the number of vehicles used and constraints (6) are for sub-tour elimination. Constraints (7) specify the demand and capacity while the arrival time and time related constraints are represented (8) and (9).

Note that the MTZ-based VRP is NP-hard with the computational complexity of $O\left(2^{n}\right)$. Accordingly, it is not an easy task to solve the VRP, especially when the size of the problem is large with hundreds or thousands of variables. This is clearly a limitation of the mathematical model, which becomes apparent if order picking routing is to be sought for a very large warehouse. However, as the focus of this paper is not on the investigation of computational complexity of VRP but on examining the potential of VRP and simulation-based routing methods for order picking, we refer readers to Toth and Vigo [32] for the computational challenge VRP poses. Indeed, VRP has been studied quite extensively, and a variety of effective heuristic algorithms, e.g., the insertion method in conjunction with tabu search [21], simulated annealing [24], and brand-and-cut [22], have been developed, which can be easily applied to solving our problem.

Note also that the route obtained by solving the mathematical optimization model may not be intuitive to order pickers because it will not have structured rules that other routings policies have. In fact, the names for other policies such as S-shape, return, mid-point, and largest gap represent the routing rules order pickers follows. This issue, however, may be easily resolved with the aid of portable IT-enabled devices that can tell directions to order pickers. Such portable devices with the real-time navigation may be attached to the material handling machines such as forklifts.

\subsection{Distance matrix}

The distance between a pair of nodes is provided by means of a distance matrix. The shortest distance between any two nodes is calculated as follows. Let $x$ be the across aisle coordinate and $z$ be the coordinate along the aisle as shown in Fig. 6. If $x_{1}=x_{2}$, i.e., the two nodes are located in the same aisle, the distance between the two nodes can be easily calculated: $t_{12}=z_{2}-z_{1}$ where $t_{12}$ is the distance between node 1 and 2 . Otherwise, the distance between the two nodes can be calculated as follows [23]:

$$
\begin{aligned}
t_{12}= & \min \left(\left|z_{1}-B\right|+\left|x_{2}-x_{1}\right|+\left|z_{2}-B\right|,\right. \\
& \left|z_{1}-M\right|+\left|x_{2}-x_{1}\right|+\left|z_{2}-M\right|, \\
& \left.\left|z_{1}-T\right|+\left|x_{2}-x_{1}\right|+\left|z_{2}-T\right|\right)
\end{aligned}
$$




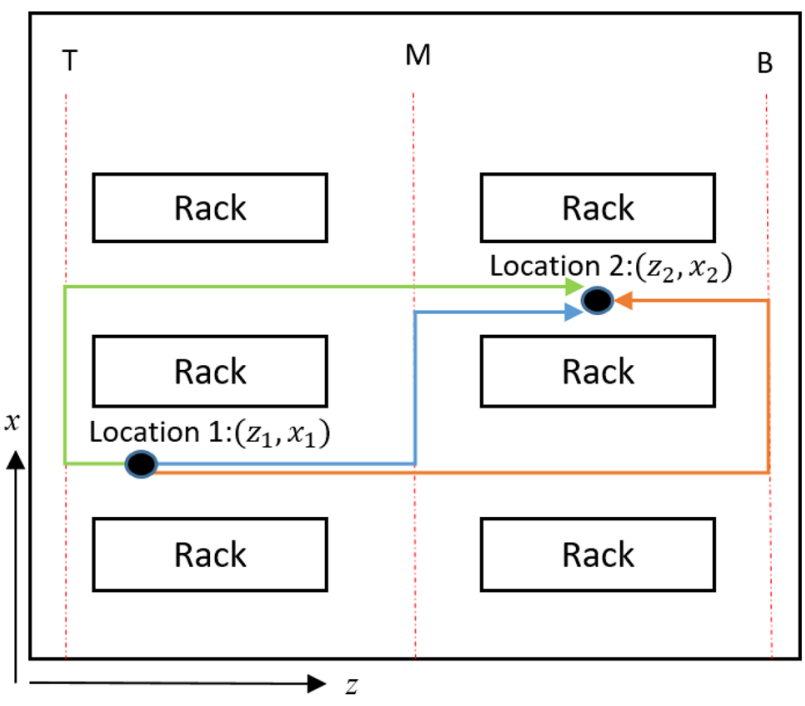

Fig. 6 Distance between two nodes. Source: [23]

where $T, M$, and $B$ are the $z$-coordinate of top cross aisle, middle cross aisle, and bottom cross aisle, respectively. The top cross aisle, middle cross aisle, and bottom cross aisle locations are shown in Fig. 6. The distance matrix calculated this way is used for the optimization model, alternative policies, and simulation.

\subsection{Alternative routing policies}

The objective of the vehicle routing model is to minimize the travel distance/time. It is important to note that the optimal shortest route may not be the most frequently used one by order pickers in practice. This is mainly because the optimal route may not be readily available to order pickers when they start a tour, which consists of the set of pick locations and associated routes, for order picking. Moreover, the optimal shortest route for each tour will be different, which may be not very convenient for order pickers because it is in general easier to follow certain consistent rules instead of following different routes for each tour. Finally, the vehicle routing problem needs to be solved for each order picking tour, which could be time consuming that may require a high level of computing power. For those reasons, other routing policies such as S-shape, return, mid-point, and largest gap, which will be briefly introduced in this section, have been employed.

However, the recent rapid development of information technology including Internet of Things (IOT) and portable/mobile electronic devices, state-of-the-art computing power, real-time location tracking techniques, and enhanced heuristic algorithms make it possible to provide a real-time routing and guidance information for order pickers through the navigation device embedded or attached to the material handling equipment. Hence, it is important to compare traditional intuitive routing policies with the vehicle routing-based shortest path routing, and evanluate the shortest path routing policy. In order to achieve this, a simulation model is built in which two order demand scenarios (one with uniform distribution and the other with more pick-ups assigned to the areas close to drop-off location) are considered. Before we proceed further, the traditional routing policies such as S-shape, return, mid-point and largest gap, illustrated in Fig. 10 where pick locations are shown as blue boxes, are briefly introduced in this paper.

\subsubsection{S-shape}

In the S-shape policy, the path followed by the order pickers is in the shape of an S. This implies that any aisle containing at least one pick is traveled entirely by the order picker as illustrated in Fig. 7a. Aisles without picks are not entered and the order picker returns to the drop-off location (depot) from the last visited aisle.

\subsubsection{Return}

In the return policy, an aisle is entered and exited from the same end as shown in Fig. 7b. Only aisles with picks are entered. If most of the pick locations are on one end of each aisle, this method can be quite effective. For example, in a warehouse where a class-based storage method is implemented and the highest-velocity items are placed at the front of each aisle, it is likely that return routing will be a favorable policy.

\subsubsection{Mid-point}

In the mid-point policy, the warehouse is divided into two halves. Picks from the bottom half are retrieved from the bottom cross aisle, while picks from the top half are retrieved from the top cross aisle, which is illustrated in Fig. 7c. If the number of picks per aisle are small, this policy provides better results than the S-shape policy [23].

\subsubsection{Largest gap}

In the largest gap policy, order pickers avoid the largest gap during the picking operation. When there is a pick up location in an aisle, there can be three gaps: (1) the distance between the top cross aisle and the first pick location in the aisle, (2) the distance between two middle pick locations, and (3) the distance between the bottom cross 


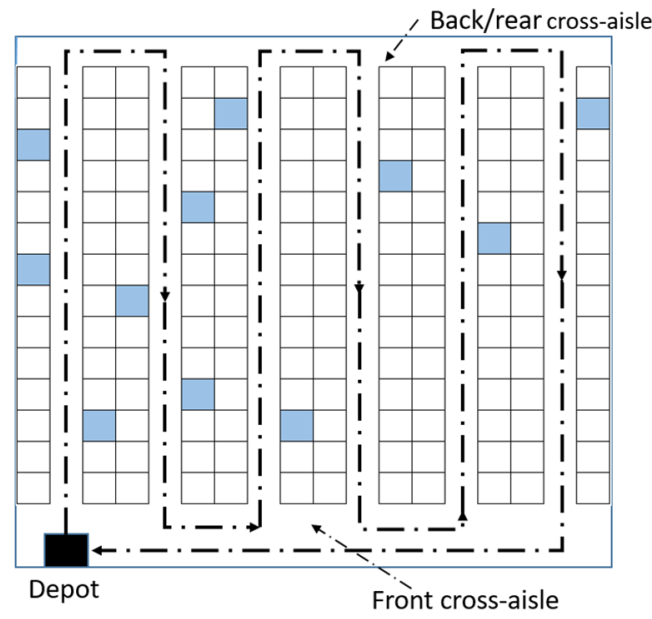

(a) S-Shape

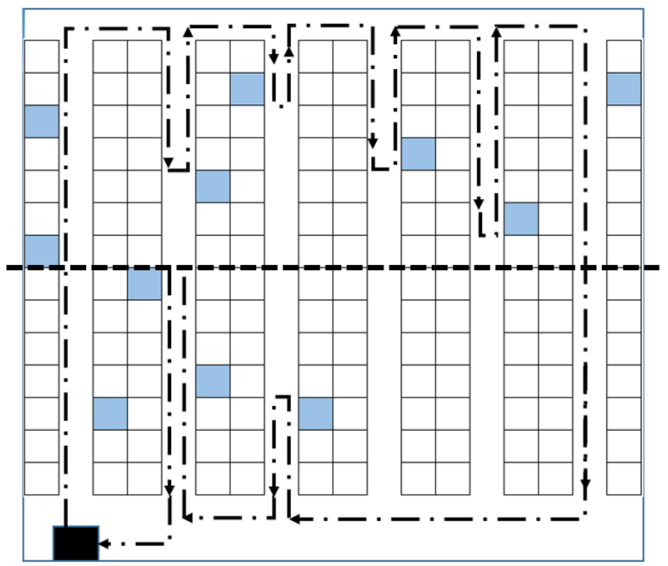

(c) Mid-Point

Fig. 7 Alternative policies. Revised from De Koster et al. [9]

aisle and the last pick location. The largest of these three gaps is avoided as shown in Fig. $7 d$.

\subsection{Simulation model}

The effectiveness of the shortest picking route is evaluated and compared with other routing policies using simulation. In particular, two random demand scenarios are considered: one with the uniform demand distribution throughout the warehouse and the other with differentiated demand distribution (half of the warehouse has more demand than the other half). This is analogous to the two storage policies: random and zone storage policies, which are frequently used in practice.

Visual Basic for Applications (VBA), a programming language embedded in Microsoft Excel, is used for simulation due to its ability of building user defined functions (for more flexibility), automating processes and ease of access,

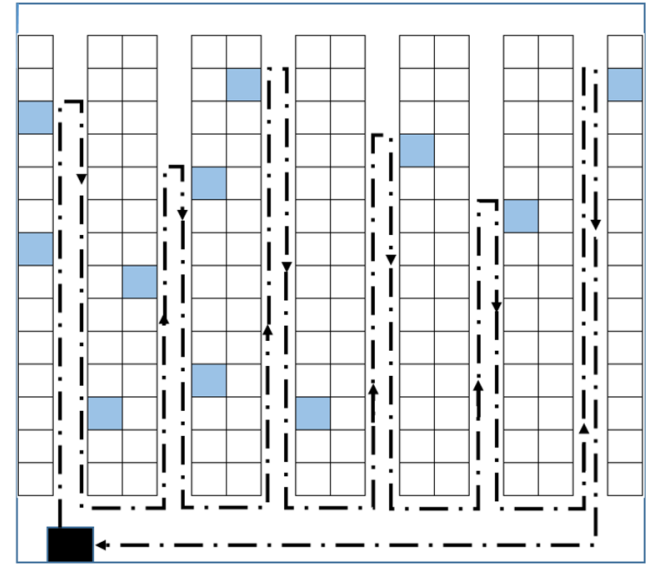

(b) Return

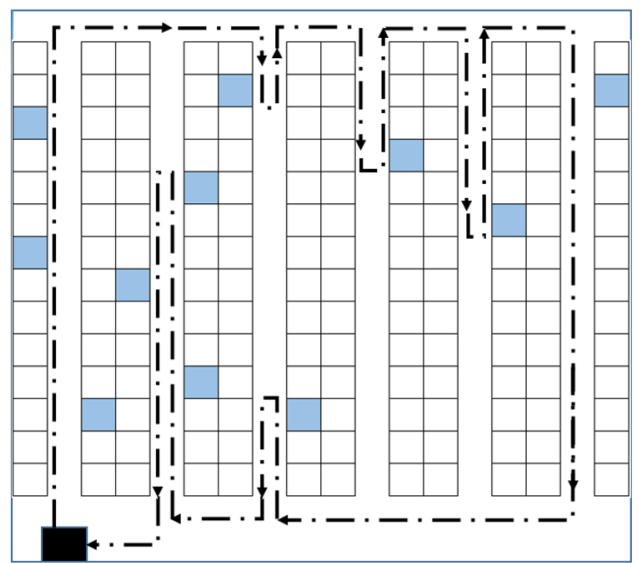

(d) Largest Gap and integration with the database. The following sections explain the development and working of the simulation model developed using VBA.

\subsubsection{Warehouse layout and distance matrix development}

This section explicates the warehouse layout and development of the distance matrix. The data has been collected by physically inspecting and measuring the layout of the partner company's actual warehouse rack sections. In the warehouse considered in this paper, there are 450 bay locations. The layout of the warehouse (rack section) shown in Fig. 4 is transferred into a 2-dimensional graph with the vertical axis $x$ and the horizontal axis $z$. For the sake of simplicity, the height of the rack is ignored. The $x$ axis denotes the across aisle coordinate while the $z$ axis represents the coordinate along the aisles (see Fig. 6). Each bay is assigned with $x$ and $z$ 
coordinates, considering its actual locations and dimensions. These coordinates enable the creation of a distance matrix for each pair of nodes (bay locations), which plays an important role in the mathematical model and simulation. The sample distance matrix for 15 locations is shown in Fig. 8.

\subsubsection{Scenario description}

To evaluate the routing policies by simulation, the inherent random pick locations and random pick amount need to be considered for which two scenarios are generated. In scenario 1 , it is assumed that the pick locations are uniformly distributed throughout the racks section of the warehouse as shown in Fig. 9a, which may correspond to the random storage policy. In scenario 2 , the warehouse is divided into two sections as shown in Fig. 9b, which may correspond to the zone storage policy. The first section (left section) is near to the inbound area and has less frequency items whereas the second section (right section) is close to the outbound area and holds high frequency items. As the high frequency items are placed near the outbound area, $75 \%$ of pick locations are generated from the right section while $25 \%$ of them are from the left section. As such, the number of picks generated on the right section near the outbound area are three times more than that of the left section near the inbound area. Note that within each section, the pick locations are uniformly distributed.

In each scenario, 14 pick locations are generated. Including the depot (drop-off location), there are a total of 15 locations. In scenario 1, the 14 locations are uniformly distributed among the 450 location points (bays), which implies that each location has an equal probability to be chosen. The size of demand (number of items to be picked) at each location is also randomly generated between 1 and 2, such that the total number of items for a single tour does not exceed the vehicle capacity that is assumed to be 30. In scenario 2, a pick location is generated in two steps. In the first step, either left or right section of the racks is

\begin{tabular}{|c|c|c|c|c|c|c|c|c|c|c|c|c|c|c|c|c|c|}
\hline & & Pick Locations & 0 & 181 & 86 & 267 & 247 & 238 & 235 & 193 & 223 & 212 & 233 & 215 & 395 & 235 & 113 \\
\hline & & $z$ & 374.5 & 124.75 & 187.25 & 262.25 & 324.75 & 212.25 & 174.75 & 274.75 & 337.25 & 199.75 & 149.75 & 237.25 & 299.75 & 174.75 & 212.25 \\
\hline & & $\mathrm{x}$ & 2.5 & 119.5 & 54.75 & 174.25 & 156 & 156 & 156 & 119.5 & 137.75 & 137.75 & 156 & 137.75 & 257.25 & 156 & 64.75 \\
\hline$z$ & $x$ & Locations & 0 & 181 & 86 & 267 & 247 & 238 & 235 & 193 & 223 & 212 & 233 & 215 & 395 & 235 & 113 \\
\hline 374.5 & 2.5 & 0 & 0 & 366.75 & 239.5 & 284.5 & 203.75 & 316.25 & 353.25 & 217.25 & 173 & 310 & 378.25 & 273 & 330 & 353.25 & 225 \\
\hline 124.75 & 119.5 & 181 & 366.75 & 0 & 152.25 & 192.25 & 236.5 & 124 & 136.5 & 150 & 230.75 & 93.25 & 161.5 & 130.75 & 312.75 & 136.5 & 142.25 \\
\hline 187.25 & 54.75 & 86 & 239.5 & 152.25 & 0 & 194.5 & 238.75 & 126.25 & 138.75 & 152.25 & 233 & 95.5 & 163.75 & 133 & 315 & 138.75 & 35 \\
\hline 262.25 & 174.25 & 267 & 284.5 & 192.25 & 194.5 & 0 & 180.75 & 93.25 & 105.75 & 192.25 & 186.5 & 99 & 130.75 & 136.5 & 245.5 & 105.75 & 184.5 \\
\hline 324.75 & 156 & 247 & 203.75 & 236.5 & 238.75 & 180.75 & 0 & 137.5 & 150 & 186.5 & 105.75 & 143.25 & 175 & 180.75 & 226.25 & 150 & 228.75 \\
\hline 212.25 & 56 & 238 & 316.25 & 124 & 126.25 & 93.25 & 137.5 & 0 & 37.5 & 124 & 168.25 & 30.75 & 62.5 & 68.25 & 213.75 & 37.5 & 116.25 \\
\hline 174.75 & 156 & 235 & 353.25 & 136.5 & 138.75 & 105.75 & 150 & 37.5 & 0 & 136.5 & 180.75 & 43.25 & 75 & 80.75 & 226.25 & 0 & 128.75 \\
\hline 274.75 & 119.5 & 193 & 217.25 & 150 & 152.25 & 192.25 & 186.5 & 124 & 136.5 & 0 & 155.75 & 93.25 & 161.5 & 130.75 & 312.75 & 136.5 & 142.25 \\
\hline 337.25 & 7.75 & 223 & 173 & 230.75 & 233 & 186.5 & 105.75 & 168.25 & 180.75 & 155.75 & 0 & 137.5 & 205.75 & 175 & 232 & 180.75 & 223 \\
\hline 199.75 & 137.75 & 212 & 310 & 93.25 & 95.5 & 99 & 143.25 & 30.75 & 43.25 & 93.25 & 137.5 & 0 & 68.25 & 37.5 & 219.5 & 43.25 & 85.5 \\
\hline 149.75 & 156 & 233 & 378.25 & 161.5 & 163.75 & 130.75 & 175 & 62.5 & 75 & 161.5 & 205.75 & 68.25 & 0 & 105.75 & 251.25 & 75 & 153.75 \\
\hline 237.25 & 137.75 & 215 & 273 & 130.75 & 133 & 136.5 & 80.75 & 68.25 & 80.75 & 130.75 & 175 & 37.5 & 105.75 & 0 & 257 & 80.75 & 123 \\
\hline 299.75 & 257.25 & 395 & 330 & 312.75 & 315 & 245.5 & 226.25 & 213.75 & 226.25 & 312.75 & 232 & 219.5 & 251.25 & 257 & 0 & 226.25 & 305 \\
\hline 174.75 & 156 & 235 & 353.25 & 136.5 & 138.75 & 105.75 & 150 & 37.5 & 0 & 136.5 & 180.75 & 43.25 & 75 & 80.75 & 226.25 & 0 & 128.75 \\
\hline 212.25 & 64.75 & 113 & 225 & 142.25 & 35 & 184.5 & 228.75 & 116.25 & 128.75 & 142.25 & 223 & 85.5 & 153.75 & 123 & 305 & 128.75 & 0 \\
\hline
\end{tabular}

Fig. 8 Sample distance matrix

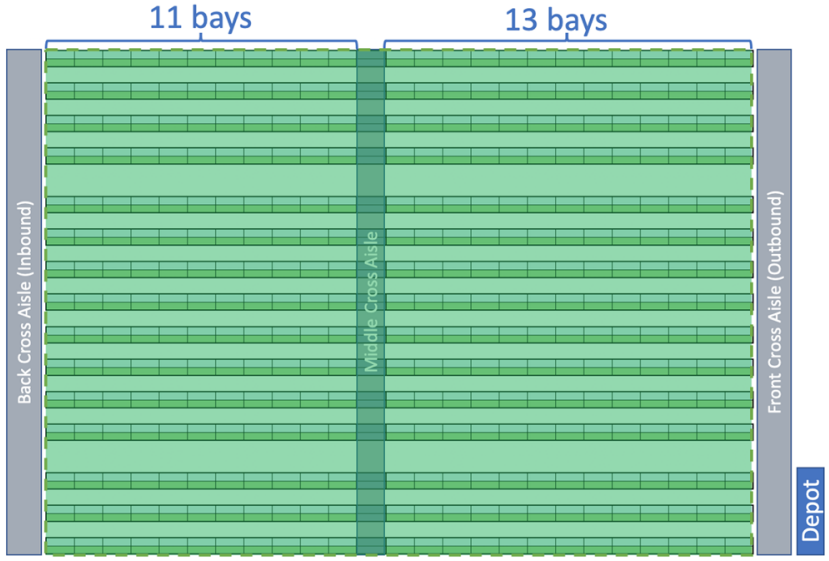

(a) Scenario 1
11 bays

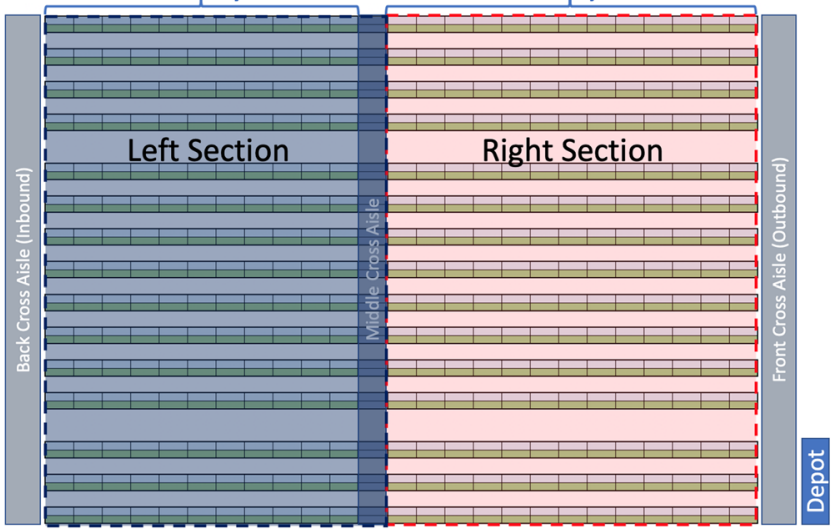

(b) Scenario 2

Fig. 9 Two scenarios 
chosen with the probability of $75 \%$ and $25 \%$, respectively. Then, a pick location is chosen based on the uniform distribution within the selected section. This process is repeated until all pick locations are generated. The number of items to be picked (size of demand) is still randomly distributed between 1 and 2 for each pick location, such that the vehicle capacity is never exceeded.

\subsubsection{Vehicle routing model and alternative policies}

The VRP has been solved using the programming language Julia in conjunction with the off-the-shelf solver Gurobi. The distance and demand matrices are used as an input to this model. Once the model is implemented and an optimal solution is found, the total distance traveled on the route is calculated. As it is assumed that the average speed of material handling equipment is 5 miles per hour, which is determined based on the discussion with the partner company's warehouse manager, the total travel time can also be calculated.

Next, for the same pick locations and demand quantities, the distances traveled for the alternative policies are calculated. These include S-shape, return, mid-point and largest gap. The logic for each policy is developed and coded by means of VBA. For the S-shape policy, any aisle containing at least one pick is traversed entirely. Aisles with no picks are not entered. Order pickers return to the depot after the last visited aisle. For the return policy, order pickers enter and leave the aisle from the same side of the aisle. Only the aisles with pick locations are entered and each aisle is traversed as far as the last pick location in the aisle. For the mid-point policy, warehouse is divided into two areas. Picks in the front half are accessed from the front cross aisle, while picks in the back half are accessed by the back cross aisle. The picks in the middle cross aisle are accessed from the front cross aisle. Finally, for the largest gap policy, the order pickers avoid the largest gap during the picking operation. The distances are then translated into travel time in the aforementioned manner.

\subsection{Model validation and verification}

After the formulation of the problem, data collection, and model development, the next essential steps of a simulation study are model verification and validation. The assumptions document is examined to ensure valid the representation of the system, which has been carried out by discussions with the warehouse manager and a careful study and analysis of the real order picking processes. Next, the models developed on Julia and VBA, i.e., the mathematical and simulation models, respectively, have been verified by means of line-by-line debugging and trial runs with simple problems of which the answers are known. After the verification step, the models are validated to be an apt representation of the system. For this, experimental runs are carried out, and then the results are compared with the actual system outputs.

\section{Results, analyses, and discussion}

The mathematical model is solved with Julia 1.1 in conjunction with Gurobi 9.0 solver and the simulation is implemented by VBA. The implementation is done using a Mac computer with an Intel core i7 CPU @ $2.2 \mathrm{GHz}$ and 16 GB RAM. The computation time is within a reasonable range (e.g., within 15 seconds) for experiments. A customized random number generator (prime modulus multiplicative linear congruential generator) is used to generate uniform variates for pick locations. For each scenario in simulation, 30 replications are made to obtain reliable

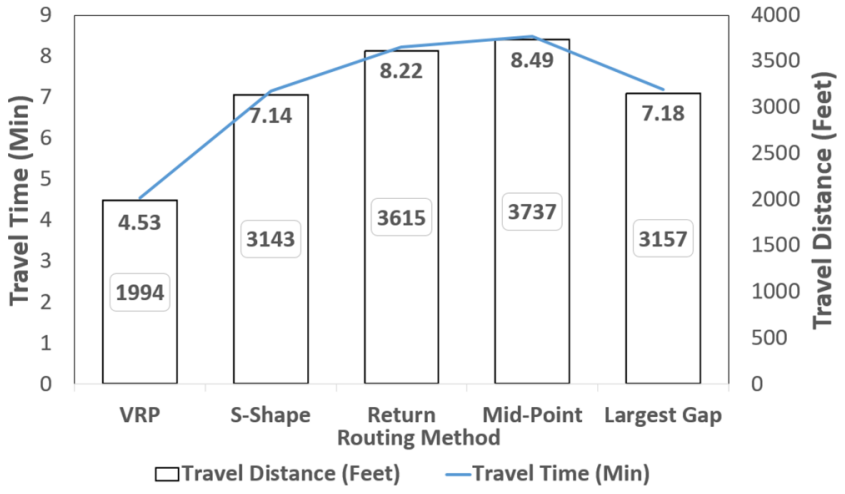

(a) Scenario 1

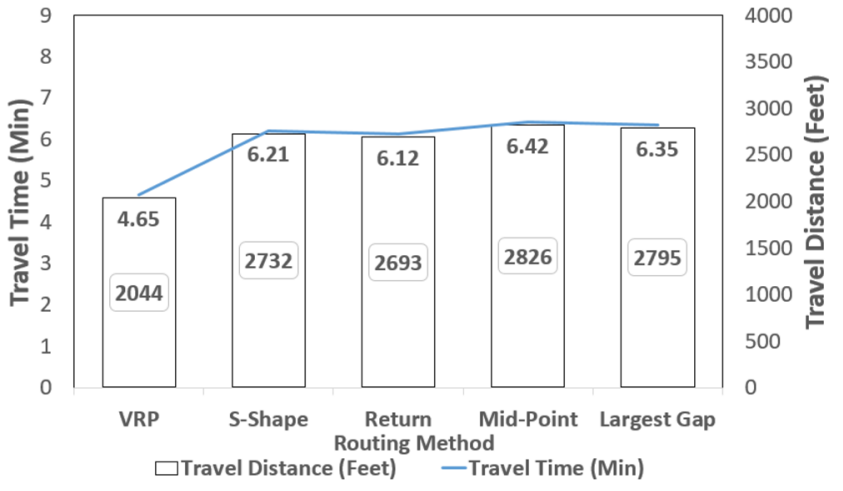

(b) Scenario 2

Fig. 10 Travel distance and time for routing policies 


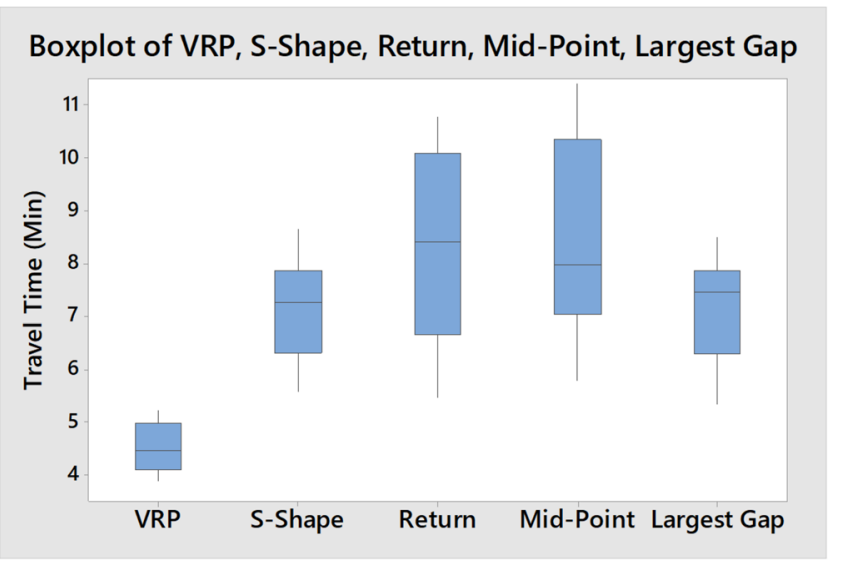

(a) Scenario 1

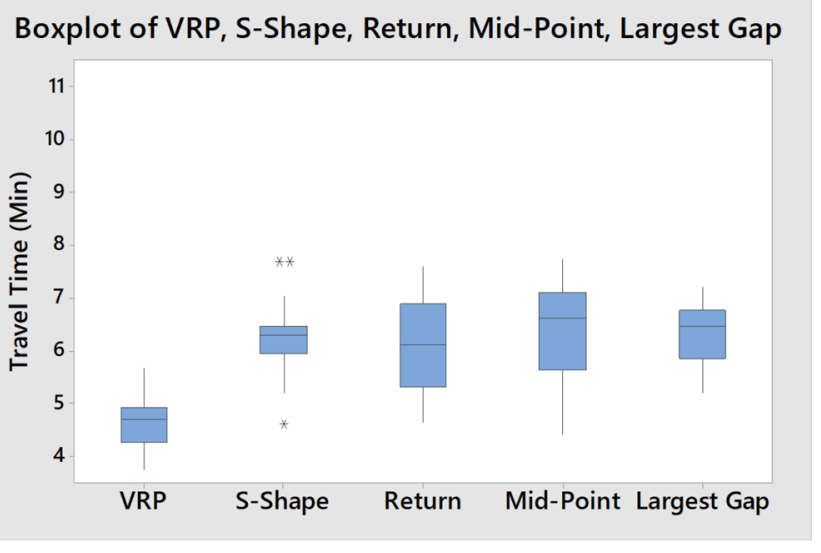

(b) Scenario 2

Fig. 11 Travel time box plot for routing policies

results and perform statistical analysis. The results are shown in Figs. 10 and 11. In particular, the average travel time and distance are shown in Fig. 10. The VRP results are $4.53 \mathrm{~min}(1994 \mathrm{ft})$ and $4.65 \mathrm{~min}(2044 \mathrm{ft})$ for scenario 1 and 2 , respectively, in terms of travel time (distance), while other routing policy results are more than $6 \mathrm{~min}$ for both scenarios. The box plots of travel time for 30 replication results are shown in Fig. 11 in which VRP travel times are significantly lowers than other routing policy travel times in both scenarios. Interestingly, the scenario 2 result for VRP policy is not significantly better than scenario 1 result while other policies show some improvements in scenario 2 over scenario 1 . More detailed analyses follow for each scenario in the subsequent sections.

\subsection{Scenario 1 results}

To analyze and compare the performance of different routing methods using statistical methods, we employ a one-way ANOVA that tests the equality of two or more means. Then, we perform a Tukey's multiple comparison test to identify which routing policy performs better than the other pairwise in terms of the average travel time. In ANOVA, the null hypothesis is that all travel time means are equal and the alternative hypothesis is that at least one mean is different. That is, $\mathrm{H}_{0}=\mu_{1}=\mu_{2}=\mu_{3}=\mu_{4}=\mu_{5}$ and $\mathrm{H}_{a}=$ At least one pair of means are different from each other where $\mathrm{H}_{0}$ and $\mathrm{H}_{a}$ represent the null and alternate hypotheses, respectively, and $\mu_{1}, \mu_{2}, \mu_{3}, \mu_{4}$, and $\mu_{5}$ are the five routing policies' population means for travel times. The significance level chosen is $5 \%$ and the equal variance assumption is taken for the analysis. The ANOVA results are summarized in Table 1.
Table 1 ANOVA results: scenario 1

\begin{tabular}{lrrrrr}
\hline Source & DF & Adj SS & Adj MS & F-Value & $p$ Value \\
\hline $\begin{array}{l}\text { Routing } \\
\quad \text { algorithms }\end{array}$ & 4 & 293.6 & 73.41 & 51.57 & 0.00 \\
Error & 145 & 206.4 & 1.424 & & \\
Total & 149 & 500.1 & & & \\
\hline
\end{tabular}

Table 2 Grouping using Tukey's test

\begin{tabular}{llllll}
\hline Routing algorithm & $\mathrm{N}$ & Mean & SD & $95 \% \mathrm{Cl}$ & Group \\
\hline Largest gap & 30 & 7.18 & 0.96 & $(6.75,7.61)$ & $\mathrm{B}$ \\
Mid-point & 30 & 8.49 & 1.62 & $(8.06,8.92)$ & $\mathrm{A}$ \\
Return & 30 & 8.22 & 1.65 & $(7.79,8.65)$ & $\mathrm{A}$ \\
S-shape & 30 & 7.14 & 0.79 & $(6.71,7.57)$ & $\mathrm{B}$ \\
VRP & 30 & 4.53 & 0.46 & $(4.10,4.96)$ & $\mathrm{C}$ \\
\hline
\end{tabular}

The decision-making process for a hypothesis test is based on the $p$ value, which indicates the probability of falsely rejecting the null hypothesis when it is indeed true. The null hypothesis is rejected because the $p$ value is 0.00 , which implies that the average travel times are not the same for different routing policies.

To assign the routing policies to the group of the similar travel times, the Tukey's test is conducted, which is shown in Table 2. The five routing polices can be divided into three groups, denoted by $A, B$, and $C$ where each group has statistically similar polices with respect to their average travel time. In Table 2, mid-point and return polices belong to the same group A while largest gap and S-shape are in the $B$ group. Note that the VRP is the only one that is in group $C$, which implies that VRP is significantly different than other polices. Indeed, VRP outperforms all the 
Fig. 12 Tukey's test: scenario 1

Table 3 Tukey simultaneous tests for differences of means
Tukey Simultaneous $95 \%$ Cls

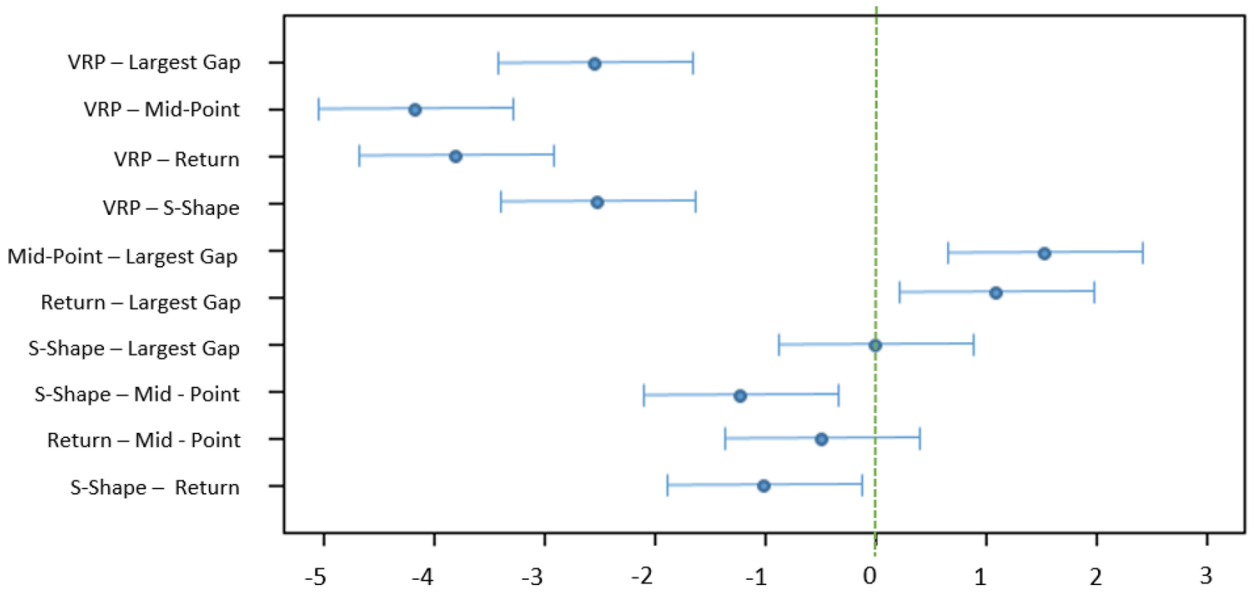

\begin{tabular}{lclcrl}
\hline Difference of levels & $\begin{array}{l}\text { Difference of } \\
\text { means }\end{array}$ & SE of difference & $95 \% \mathrm{Cl}$ & T-Value & $p$ Value \\
\hline VRP-Largest gap & -2.64 & 0.31 & $(-3.50,-1.79)$ & -8.58 & 0 \\
VRP-Mid-point & -3.96 & 0.31 & $(-4.81,-3.11)$ & -12.86 & 0 \\
VRP-Return & -3.69 & 0.31 & $(-4.54,-2.83)$ & -11.96 & 0 \\
VRP-S-shape & -2.61 & 0.31 & $(-3.46,-1.76)$ & -8.48 & 0 \\
Mid-point-Largest gap & 1.32 & 0.31 & $(0.46,2.17)$ & 4.27 & 0 \\
S-shape-Largest gap & -0.03 & 0.31 & $(-0.88,0.82)$ & -0.11 & 1 \\
Return-Mid-point & -0.28 & 0.31 & $(-1.13,0.57)$ & -0.9 & 0.90 \\
S-shape-Mid-point & -1.35 & 0.31 & $(-2.20,-0.50)$ & -4.38 & 0 \\
Return-Largest gap & 1.04 & 0.31 & $(0.19,1.89)$ & 3.38 & 0.01 \\
S-shape-Return & -1.07 & 0.31 & $(-1.92,-0.22)$ & -3.48 & 0.01 \\
\hline
\end{tabular}

other routing methods, which can be verified from pairwise Tukey's test summarized in Table 3 and Fig. 12. The pairwise Tukey's test has the null hypothesis: $\mathrm{H}_{0}: \mu_{i}=\mu_{j}$, and the alternative hypothesis: $\mathrm{H}_{a}: \mu_{i} \neq \mu_{j}$ where $i$ and $j$ are indexes for routing policies. If the $p$ value of the test pair is less then 0.05 (at the significance level of 5\%), then we reject the null hypothesis, otherwise not. As shown in Table 3 and Fig. 12, the VRP result is significantly less than largest gap, mid-point, return, and S-shape. In addition, the largest gap shows the worst results compared to other routing policies. In summary, VRP is the best and largest gap is the worst in scenario 1.

\subsection{Scenario 2 results}

Similar to Sect. 4.1, ANOVA and Tukey's test are used to compare different routing policies in terms of the travel time for scenario 2 . The same hypotheses and significance level are used. The ANOVA results are summarized in Table 4. Consistent with scenario 1, the null hypothesis
Table 4 ANOVA results

\begin{tabular}{lrrrrr}
\hline Source & DF & Adj SS & Adj MS & F-Value & $p$ Value \\
\hline $\begin{array}{l}\text { Routing } \\
\quad \text { algorithms }\end{array}$ & 4 & 65.41 & 16.35 & 31.76 & 0.00 \\
Error & 145 & 74.67 & 0.52 & & \\
Total & 149 & 1140.08 & & & \\
\hline
\end{tabular}

Table 5 Grouping using Tukey test

\begin{tabular}{llllll}
\hline Routing algorithm & $\mathrm{N}$ & Mean & $\mathrm{SD}$ & $95 \% \mathrm{Cl}$ & Grouping \\
\hline Largest gap & 30 & 6.35 & 0.57 & $(6.09,6.61)$ & $\mathrm{A}$ \\
Mid-point & 30 & 6.42 & 0.87 & $(6.61,6.68)$ & $\mathrm{A}$ \\
Return & 30 & 6.12 & 0.89 & $(5.86,6.38)$ & $\mathrm{A}$ \\
S-shape & 30 & 6.21 & 0.68 & $(5.95,6.47)$ & $\mathrm{A}$ \\
VRP & 30 & 4.65 & 0.49 & $(4.39,4.91)$ & $\mathrm{B}$ \\
\hline
\end{tabular}


Fig. 13 Tukey's pairwise test: scenario 2

Table 6 Tukey simultaneous tests for differences of means
Tukey Simultaneous $95 \% \mathrm{Cls}$

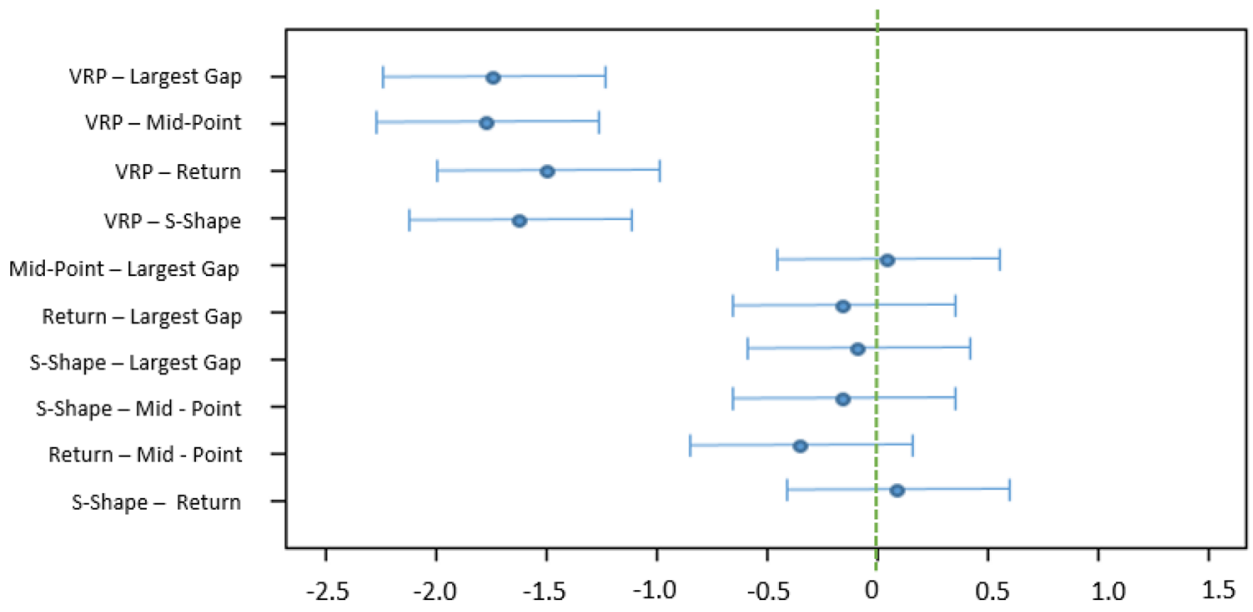

\begin{tabular}{llllrl}
\hline Difference of levels & $\begin{array}{l}\text { Difference of } \\
\text { means }\end{array}$ & SE of difference & $95 \% \mathrm{Cl}$ & T-Value & $p$ Value \\
& -1.71 & 0.18 & $(-2.22,-1.19)$ & -9.21 & 0 \\
VRP-Largest gap & -1.77 & 0.18 & $(-2.29,-1.26)$ & -9.58 & 0 \\
VRP-Mid-point & -1.47 & 0.18 & $(-1.99,-0.96)$ & -7.96 & 0 \\
VRP-Return & -1.56 & 0.18 & $(-2.07,-1.05)$ & -8.43 & 0 \\
VRP-S-shape & 0.07 & 0.18 & $(-0.44,0.58)$ & 0.37 & 0.99 \\
Mid-point-Largest gap & -0.23 & 0.18 & $(-0.74,0.28)$ & -1.25 & 0.72 \\
Return-Largest gap & -0.14 & 0.18 & $(-0.66,0.37)$ & -0.78 & 0.94 \\
S-shape-Largest gap & -0.21 & 0.18 & $(-0.72,0.30)$ & -1.15 & 0.78 \\
S-shape-Mid-point & -0.30 & 0.18 & $(-0.81,0.21)$ & -1.62 & 0.48 \\
Return-Mid-point & 0.09 & 0.18 & $(-0.42,0.60)$ & 0.47 & 0.99 \\
S-shape-Return & & & & &
\end{tabular}

Table 7 Sample $T$-test for VRPs

\begin{tabular}{lllll}
\hline Sample & N & Mean & SD & SE mean \\
\hline VRP (scenario 1) & 30 & 4.53 & 0.46 & 0.08 \\
VRP (scenario 2) & 30 & 4.64 & 0.49 & 0.09 \\
\hline
\end{tabular}

is rejected in scenario 2 . That is, the average travel times are not the same for different routing policies.

Furthermore, to assign the routing policies to the group of the similar travel times, Tukey's test is conducted, which is shown in Table 5. The five routing polices can be divided into two groups, denoted by $A$ and $B$ where each group has statistically similar polices with respect to their average travel time. In Table 5, midpoint, return, largest gap and S-shape are in the A group while the VRP is in group $B$, which implies that VRP is significantly different than other polices. Unlike scenario 1 , there are two groups, instead of 3 , which implies that while VRP (in group B) is significantly differnt than others, all other routing policies are indifferent to each other. VRP again outperforms all other polices, which can be verified from pairwise Tukey's test summarized in Table 6 and Fig. 13. The VRP result is significantly less than largest gap, mid-point, return, and S-shape. In summary, VRP is the best policy for scenario 2 as well as for scenario 1 (Table 7).

\subsection{Summary of results}

The simulation results are summarized in Table 8 along with comments that contain some suggestions and insights. With a 95 percent confidence level, VRP outperforms all other routing policies for both scenarios. That is, the VRP travel time is significantly smaller than that of the alternative routing policies. The average travel time for VRP is $4.53 \mathrm{~min}$ for scenario 1 and $4.64 \mathrm{~min}$ for scenario 2. S-shape and largest gap policies are the next best ones for scenario 1 but this is not the case in scenario 2. No significant differences is found between these two routing methods, with the mean time for S-shape being 7.14 
min and that for largest gap being $7.18 \mathrm{~min}$ in scenario 1. The mid-point and return routing policies are significantly more time consuming with the mean times being 8.49 and $8.22 \mathrm{~min}$, respectively, in scenario 1. However, all four polices except VRP are very similar to each other in scenario 2.

To analyze the VRP results between scenario 1 and scenaro 2, we conduct a 2-sample $t$-test to compare the time taken by the VRP policy for the two scenarios. First, the box plots for the two VRP results are drawn in Fig. 14, in which scenario 1 result shows more variability between the first and third quartiles (blue boxes). However, in scenario 2 the VRP travel time shows bigger maximum and minimum values compared to scenario 1 . This implies that the zone storage could result in some extreme travel times in some situations. In the two sample $t$-test, the null hypothesis is that the travel time means are equal between the two scenarios while

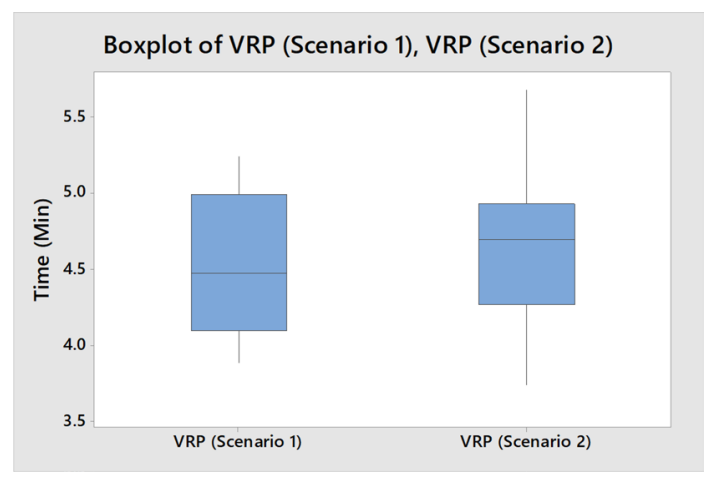

Fig. 14 VRP boxplots for two scenarios
Table 9 Sample $T$-test results

\begin{tabular}{lllll}
\hline Difference & $\mathrm{Cl}(95 \%)$ & T-Value & $\mathrm{DF}$ & $p$ value \\
\hline-0.11 & $(-0.36,0.13)$ & -0.93 & 57 & 0.35 \\
\hline
\end{tabular}

the alternative hypothesis is that the means are different. That is, $\mathrm{H}_{0}: \mu_{1}=\mu_{2}$ and $\mathrm{H}_{a}: \mu_{1} \neq \mu_{2}$. The significance level chosen is $5 \%$ and the results are summarized in Table 9. From the results, as $p$ value $>0.05$ the null hypothesis cannot be rejected, implying the two VRP results show no significant differences. We may conclude that the storage policy between random and zone methods have no statistical impacts on the VRP results while other routing policy results improve for scenario 2 in our example.

\subsection{Analyses and discussion}

It is evident that the solution vehicle routing model entails the least amount of travel time, and is significantly better than the other routing method. However, the solution of this algorithm is unique for every order and needs to be executed for each individual set of pick locations. Also, it is not intuitive for the forklift operators unless each operator is assigned a tablet or other device to view the path that is to be traveled. Even though, statistically, the largest gap routing method performs as well as the S-shape method, the largest gap method is not intuitive for the operators. It is not possible to visually determine the largest gap without the aid of mathematical calculations. Hence, the S-shape policy

Table 8 Summary of results

\begin{tabular}{|c|c|c|c|}
\hline Routing policy & $\begin{array}{l}\text { Avg. time (min) } \\
\text { (Scenario 1) }\end{array}$ & $\begin{array}{l}\text { Avg. tme (min) } \\
\text { (Scenario 2) }\end{array}$ & Comment \\
\hline Vehicle routing & $4.53($ Rank = 1) & $4.64(\operatorname{Rank}=1)$ & $\begin{array}{l}\text { Provides the shortest possible travel time } \\
\text { Additional software needed } \\
\text { Not intuitive for operators }\end{array}$ \\
\hline S-shape & $7.14($ Rank = 2) & $6.21($ Rank = 2) & $\begin{array}{l}\text { Does not provide the shortest possible travel time } \\
\text { No additional software required } \\
\text { Intuitive for operators }\end{array}$ \\
\hline Largest gap & $7.18($ Rank = 2) & $6.12($ Rank $=2)$ & $\begin{array}{l}\text { Does not provide the shortest possible travel time } \\
\text { No additional software required } \\
\text { Not intuitive for operators }\end{array}$ \\
\hline Return & $8.22($ Rank = 3) & $6.42($ Rank $=2)$ & $\begin{array}{l}\text { Time consuming } \\
\text { No additional software required } \\
\text { Intuitive for operators }\end{array}$ \\
\hline Mid-point & $8.49($ Rank = 3) & $6.35($ Rank $=2)$ & $\begin{array}{l}\text { Time consuming } \\
\text { No additional software required } \\
\text { Intuitive for operators }\end{array}$ \\
\hline
\end{tabular}


works better because of its intuitive nature. The return and mid-point routing methods are the least preferred methods because of the additional travel time. Hence, the vehicle routing model is recommended since its provides the optimal solution. However, in certain situations, it is not possible to use the vehicle routing model due to software/manpower limitations. In this case the $\mathrm{S}$-shape method is recommended.

\section{Conclusion and future work}

Order picking is one of the most critical, time restrained activity of warehouse operations. It has the ability to impact the entire supply chain. This study provides a solution to the routing optimization problem for order picking in a rectangular, multiparallel-aisle warehouse with cross aisles at the middle and end of every aisle. The objective of this study is to propose a routing policy that minimizes the total travel time/distance in the order picking process, which in turn minimizes the overall operating costs. This was achieved by means of optimization and simulation, followed by statistical analysis.

First, a mathematical model was developed as a special case of the vehicle routing problem. This model was implemented using Julia in conjuction with Gurobi. Next, a simulation model was generated using VBA based on the actual warehouse layout. Two scenarios based on the picking demand distribution within the warehouse were simulated. For a set of 15 pick locations, the simulation delivered routes for multiple routing policies: VRP, S-shape, return, mid-point, and largest gap for both scenarios. For both the scenarios, VRP outperforms the other routing methods, which was validated using ANOVA and Tukey's test. The results show that the proposed VRP based approach outperforms the S-shape, return, midpoint, largest gap methods by $36.55 \%, 44.89 \%, 46.64 \%$, and $36.9 \%$, respectively, for scenario 1 , and by $25.12 \%$, $24.01 \%, 27.57 \%$, and $26.77 \%$, respectively, for scenario 2 .

Future work can be directed towards overcoming the limitations of the current model. The simulation scenario can be modified for pick locations to accommodate more realistic situation. For this, actual data from the warehouse can be used and the state-of-the-art data analysis techniques can be used. Furthermore, heuristic algorithms can be developed to tackle more complex, realistic situations such as multiple pickers/vehicles in a large warehouse. In addition, uncertain factors such as traffic jam, errors in Kanban triggers can be considered by means of stochastic optimization. A VRP solution can be different if the capacity of order pickers is considered. Even though the capacity constraints are not considered in this paper to focus on the main topic of examining the VRP-based method for order picking routing with the aid of simulation, it will be a valuable direction to be sought in the future work.

\section{Compliance with ethical standards}

Conflict of interest The author(s) declare that they have no competing interests.

\section{References}

1. Bartholdi J, Hackman S (2014) Warehouse and distribution science: release 0.96 . The supply chain and logistics institute. Georgia Institute of Technology, Atlanta

2. Calvi K, Halawa F, Economou M, Kulkarni R, Havens R (2019) A simulation study integrated with activity-based costing for an electronic device refurbishing system. Int J Adv Manuf Technol 103:127

3. Caron F, Marchet G, Perego A (1998) Routing policies and coibased storage policies in picker-to-part systems. Int J Prod Res 36(3):713-732

4. Carvalho H, Barroso AP, Machado VH, Azevedo S, Cruz-Machado V (2012) Supply chain redesign for resilience using simulation. Comput Ind Eng 62(1):329-341

5. Çeven E, Gue KR (2015) Optimal wave release times for order fulfillment systems with deadlines. Transp Sci 51(1):52-66

6. Chen F, Wang H, Xie Y, Qi C (2016) An aco-based online routing method for multiple order pickers with congestion consideration in warehouse. J Intell Manuf 27(2):389-408

7. Chen F, Xu G, Wei Y (2019) An integrated metaheuristic routing method for multiple-block warehouses with ultranarrow aisles and access restriction. Complexity 67:228

8. Dantzig GB, Ramser JH (1959) The truck dispatching problem. Manag Sci 6(1):80-91

9. De Koster R, Le-Duc T, Roodbergen KJ (2007) Design and control of warehouse order picking: a literature review. Eur J Oper Res 182(2):481-501

10. Dekker R, De Koster M, Roodbergen KJ, Van Kalleveen H (2004) Improving order-picking response time at ankor's warehouse. Interfaces 34(4):303-313

11. Frazelle E (2002) Supply chain strategy: the logistics of supply chain management. McGraw-Hill, New York

12. Fukasawa $R$, Longo $H$, Lysgaard J, de Aragão $M P$, Reis $M$, Uchoa E, Werneck RF (2006) Robust branch-and-cut-andprice for the capacitated vehicle routing problem. Math Progr 106(3):491-511

13. Gallien J, Weber T (2010) To wave or not to wave? Order release policies for warehouses with an automated sorter. Manuf Serv Oper Manag 12(4):642-662

14. Gu J, Goetschalckx M, McGinnis LF (2007) Research on warehouse operation: a comprehensive review. Eur J Oper Rese 177(1):1-21

15. Hall RW (1993) Distance approximations for routing manual pickers in a warehouse. IIE Trans 25(4):76-87

16. Jewkes E, Lee C, Vickson R (2004) Product location, allocation and server home base location for an order picking line with multiple servers. Comput Oper Res 31(4):623-636

17. Jiang C (2010) A reliable solver of euclidean traveling salesman problems with microsoft excel add-in tools for small-size systems. JSW 5(7):761-768 
18. Juan A, Grasman S, Faulin J, Riera D, Méndez C, Ruiz B (2009) Applying simulation and reliability to vehicle routing problems with stochastic demands. In: Proceedings of the XI conference of the AllA. Citeseer, pp 201-214

19. Kleijnen JP (2005) Supply chain simulation tools and techniques: a survey. Int J Simul Process Model 1(1-2):82-89

20. Lee I, Chung S, Yoon S (2019) Two-stage storage assignment to minimize travel time and congestion for warehouse order picking operations. Comput Ind Eng 9:29

21. Li Y, Chung SH (2019) Disaster relief routing under uncertainty: a robust optimization approach. IISE Trans 51(8):869-886

22. Lysgaard J, Letchford AN, Eglese RW (2004) A new branch-andcut algorithm for the capacitated vehicle routing problem. Math Progr 100(2):423-445

23. Mohr CM (2014) Optimization of warehouse order-picking routes using vehicle routing model and genetic algorithm. State University of New York, Binghamton

24. Osman IH (1993) Metastrategy simulated annealing and tabu search algorithms for the vehicle routing problem. Ann Oper Res 41(4):421-451

25. Petersen CG (1997) An evaluation of order picking routeing policies. Int J Oper Prod Manag 17(11):1098-1111

26. Petersen CG, Aase G (2004) A comparison of picking, storage, and routing policies in manual order picking. Int J Prod Econ 92(1):11-19

27. Petersen CG, Schmenner RW (1999) An evaluation of routing and volume-based storage policies in an order picking operation. Decis Sci 30(2):481-501
28. Ratliff HD, Rosenthal AS (1983) Order-picking in a rectangular warehouse: a solvable case of the traveling salesman problem. Oper Res 31(3):507-521

29. Roodbergen KJ, Koster R (2001) Routing methods for warehouses with multiple cross aisles. Int J Prod Res 39(9):1865-1883

30. Scholz A, Schubert D, Wäscher G (2017) Order picking with multiple pickers and due dates-simultaneous solution of order batching, batch assignment and sequencing, and picker routing problems. Eur J Oper Res 263(2):461-478

31. Theys C, Bräysy O, Dullaert W, Raa B (2010) Using a TSP heuristic for routing order pickers in warehouses. Eur J Oper Res 200(3):755-763

32. Toth P, Vigo D (2014) Vehicle routing: problems, methods, and applications. SIAM, New Delhi

33. Toyota Material Handling (2016) Toyota material handling European website. https://toyota-forklifts.eu/our-offer/produ ct-range/automated-solutions/. Accessed 27 August 2016

34. Wang $Z$, Lin $L$ (2013) A simulation-based algorithm for the capacitated vehicle routing problem with stochastic travel times. J Appl Math 2013:127156

35. Wu D, Olson DL (2008) Supply chain risk, simulation, and vendor selection. Int J Prod Econ 114(2):646-655

36. Zhang $Y$ (2016) Correlated storage assignment strategy to reduce travel distance in order picking. IFAC Pap OnLine 49(2):30-35

Publisher's Note Springer Nature remains neutral with regard to jurisdictional claims in published maps and institutional affiliations. 